\title{
Effects of celecoxib augmentation of antidepressant or anxiolytic treatment on affective symptoms and inflammatory markers in patients with anxiety disorders: exploratory study Hesham Y. Elnazer ${ }^{\mathrm{a}, \mathrm{b} *}$, Anthony P. Sampson ${ }^{\mathrm{c}}$ and David S. Baldwin ${ }^{\mathrm{c}, \mathrm{d}, \mathrm{e}}$
}

\begin{abstract}
Prolonged stress has been associated with elevated levels of circulating proinflammatory cytokines. Cyclo-oxygenase-2 inhibitors such as celecoxib exert anti-inflammatory effects and may enhance the response to antidepressant drug treatment in patients with depressive disorders, but their effect on anxiety symptoms in patients with anxiety disorders is uncertain. Patients with a primary diagnosis of an anxiety disorder, with stabilised symptoms, underwent either 6 weeks of celecoxib augmentation of continued treatment $(n=18)$ or continued 'treatment as usual' $(n=9)$. Assessments included the Warwick-Edinburgh mental well-being Scale (WEMWEBS), Hospital Anxiety and Depression Scale (HADS), Oxford questionnaire of emotional side effects of antidepressants (OQUESA) and Clinical Global Impression of IIIness Severity (CGIS). Venous blood samples were collected for assays of inflammatory cytokines. Patients who underwent celecoxib augmentation showed significant reductions in anxiety (HADS-A -3.17) and depressive (HADS-D -2.11) symptoms and in overall illness severity (CGI-S -1.11), and improvements in mental well-being (WEMWBS 7.5) and positive changes in emotional responsiveness (OQUESA-RP - 3.56; OQUESA-AC
\end{abstract}

\section{Background}

Anxiety symptoms and disorders can develop when the physiological response to actual or perceived stress becomes impaired and leads to reduced levels of functioning (Kandel et al., 2012). Anxiety symptoms have been associated with decreased levels of C-reactive protein (CRP), tumour necrosis factor- $\alpha$ (TNF- $\alpha$ ), certain interleukins (IL-1b, IL-6, IL-8) and neurotrophins (Zorrilla et al., 1994; Janelidze et al., 2015; Martin et al., 2015; Priya et al., 2016). Somatic anxiety symptoms have been associated with higher levels of CRP, IL-6 and TNF- $\alpha$, whereas psychological anxiety symptoms have been associated with increased levels of CRP (Duivis et al., 2013). Furthermore, low IL-6 levels predicted symptom resolution in a non-clinical sample of participants with psychological distress (Virtanen et al., 2015). However, not all evidence is consistent; for example, a large study ( $n=1037)$ found no correlation between anxiety symptoms and inflammatory markers, after adjusting for environmental and other health factors (Baune et al., 2012).
-4.22): these were not seen with 'treatment as usual'. There were no significant changes in blood levels of inflammatory cytokines in either group. Celecoxib augmentation appeared associated with beneficial effects on anxiety and depressive symptoms and mental well-being. The findings from this pilot study merit further exploration within a double-blind, randomised placebo-controlled study. Int Clin Psychopharmacol 36: 126-132 Copyright (c) 2021 Wolters Kluwer Health, Inc. All rights reserved.

International Clinical Psychopharmacology 2021, 36:126-132

Keywords: anxiety disorders, celecoxib, COX-2 inhibitor, inflammation, nonsteroidal anti-inflammatory drugs

*data acquisition and analysis undertaken whilst based at University of Southampton a Sussex Partnership NHS Foundation Trust, Chichester, ${ }^{\mathrm{b}}$ Brighton and Sussex Medical School, University of Sussex, Brighton, ${ }^{\circ}$ Clinical and Experimental Sciences, Faculty of Medicine, University of Southampton and ${ }^{\mathrm{d} M o o d}$ Disorders Service, Southern Health NHS Foundation Trust, Southampton, UK eUniversity Department of Psychiatry and Mental Health, University of Cape Town, South Africa

Correspondence to Hesham Y. Elnazer, MD, MBBCh, MRCPsych, Bedale Medical Centre, East Sussex CMHT, Bognor Regis PO12 2SF, UK Tel: + 440300304 0400; e-mail: elnazer@gmail.com

Received 8 January 2021 Accepted 13 February 2021

A recent meta-analysis found differences in IL-10, TNF$\alpha$ and interferon-gamma (IFN- $\gamma$ ) in patients with generalised anxiety disorder (GAD), when compared to controls (Costello et al., 2019). Several published studies have detected evidence of elevated CRP, TNF- $\alpha$, IL-1 and IL-2 levels in panic disorder (Rapaport et al., 1994; Koh $e t$ al., 2004; Hoge et al., 2009; Vieira et al., 2010; Wagner et al., 2015; Glaus et al., 2017). Only few studies have examined inflammatory markers in social phobia, specific phobia and comorbid anxiety disorders with obsessive-compulsive disorder (OCD). Social phobia has been associated with decreased levels of CRP and IL-6 (Vogelzangs et al., 2013). Some studies in OCD have found decreased levels of TNF- $\alpha$ and IL-6 (Denys et al., 2004; Konuk et al., 2007; Fluitman et al., 2010), whereas others found the opposite in OCD patients with comorbid depression (Monteleone et al., 1998; Carpenter et al., 2002).

Proinflammatory and anti-inflammatory cytokines function in complementary and competing effects in producing an overall response that determines the onset, 
duration and severity of the inflammatory phase of healing. A net anti-inflammatory pattern is required for a less severe, shorter healing response (Elnazer et al., 2020). Proinflammatory cytokines can induce cyclo-oxygenase enzymes (COX) (Harden et al., 2015): COX-1 and COX-2 catalyse the oxidation of arachidonic acid to produce prostaglandin G2 (PGG2) and the peroxidation of PGG2 to prostaglandin $\mathrm{H} 2$ ( $\mathrm{PGH} 2)$, these reactions being associated with release of reactive oxygen species which can cause cell damage (Maes et al., 2012).

A meta-analysis of the influence of nonsteroidal anti-inflammatory drugs (NSAID) treatment in patients with osteoarthritis suggests potential beneficial effects on depressive symptoms (Iyengar et al., 2013). Meta-analysis of investigations of a range of anti-inflammatory treatments in patients with mood disorders found greater reductions in depressive symptom scores when compared to placebo ( 6 trials, $n=214$ ), and improved manic symptoms in patients with bipolar affective disorder (3 trials, $n=96)$ : additional analysis of investigations with celecoxib (11 randomised controlled trials) suggests it may be associated with antidepressant effects in patients with major depressive disorder, without conferring an additional side effect burden (Husain et al., 2017). A more recent randomised clinical trial found a reduction of anxiety symptoms in patients with treatment-resistant bipolar depression $(n=47)$, during celecoxib augmentation (Halaris et al., 2020). However, it remains uncertain whether celecoxib is beneficial in reducing anxiety symptoms in patients with anxiety disorders.

\section{Methods}

\section{Study sample}

Participants were recruited from general practice and secondary care mental health services. Potentially eligible patients were sent a letter with a participant information sheet, contact information return slip and stamped addressed envelope. When a potential participant contacted the research team or when a contact information slip was returned, the researcher made an appointment for clarification of study aims and procedures, and potential participants were invited to consent to a screening assessment, which included the Mini International Neuropsychiatric Interview (Sheehan et al., 1998). Patients were considered eligible for the study if they were aged between 18-70 years; had the primary diagnosis of an anxiety disorder or anxiety-related disorder, defined according to Diagnostic and Statistical Manual of Mental Disorders, 5th Edition criteria (American Psychiatric Association, 2013), and were competent to provide written consent. Patients with OCD could be included, for although OCD is now classified separately from anxiety disorders, anxiety symptoms are common and maybe the reason for seeking treatment. Patients were excluded from the study if they were outside the age range $18-70$ years, did not have a primary diagnosis of an anxiety disorder, were unable to provide written informed consent, had clinically significant alcohol or substance use in the previous three months or were pregnant or breastfeeding.

\section{Assessments and celecoxib administration}

The Hospital Anxiety and Depression Scale (HADS) (Zigmond et al., 1983) was used as the primary outcome measure. Patients were also assessed using the Clinical Global Impression of Illness Severity (CGI-S) (Guy et al., 1976), Warwick-Edinburgh Mental Well-being Scale (WEMWEBS) (Tennant et al., 2007) and Oxford questionnaire of emotional side effects of antidepressants (OQUESA) (Price et al., 2012), at Baseline and after 6 weeks of being stabilised on treatment (week 6). At week 6 , patients were invited to either undergo 6 weeks of open-label augmentation with celecoxib or continue 'treatment as usual'. Further assessments were undertaken at week 12. Treatment adherence was determined by patient report and tablet count. Venous blood samples were collected at Baseline, week 6 and 12 for determination of inflammatory marker concentrations. Exclusion criteria for celecoxib augmentation were medical contra-indication for celecoxib; history of hypersensitivity to NSAIDs; presence of cardiac impairment, thromboembolic disease, renal impairment, hepatic impairment or history of recurrent gastrointestinal ulceration; concomitant use of NSAIDs, ciclosporins, coumarins, dabigatran, ketorolac, lithium, methotrexate, phenindione, quinolones or sulfonylureas. The celecoxib dosage was $200 \mathrm{mg}$ twice daily, based on previous augmentation studies in depressed patients (Nery et al., 2008; Akhondzadeh et al., 2009; Abbasi et al., 2012).

\section{Treatment withdrawal criteria}

Participants could withdraw their consent from study procedures at any point. Treatment would be stopped immediately for any patient who experienced any celecoxib serious adverse event listed in the electronic medicines' compendium during either the preaugmentation phase or the augmentation phase. Patients were advised to access emergency help if they had shortness of breath or trouble breathing, chest pain, weakness in one part or side of the body, slurred speech, swelling of the face or throat. Patients were advised to stop celecoxib and call their general practitioner immediately if they had nausea, fatigue, pruritus, jaundice, abdominal pain, flu-like symptoms, haemoptysis, haematochezia, melena, skin rash or blisters with fever, unusual weight gain or oedematous arms, legs, hands or feet.

\section{Inflammatory marker assay and analysis Sampling}

Venous blood samples were collected from participants at Baseline and after 6 weeks of celecoxib augmentation: 8-10 $\mathrm{ml}$ samples were collected by venepuncture into a 
vacutainer containing no preservative (red top). Sampling was performed according to National Health Service procedures, observing sterile conditions, with infection control by either a nurse trained in phlebotomy or by a qualified medical practitioner.

\section{Sample preparation}

Samples were centrifuged at $1600 \mathrm{~g}$ on a Capricorn 2000 bench centrifuge for $10 \mathrm{~min}$. The plasma fraction created from the serum sample was transferred using a Pasteur pipette to suitably labelled vial tubes. Serum samples were placed in a ziplock bag and airtight sealed container inside a lockable box for initial storage at $-20^{\circ} \mathrm{C}$ then transfer to a $-80^{\circ} \mathrm{C}$ freezer for longer-term storage.

\section{Quantification}

Luminex multianalyte profiling (xMAP) technology employs proprietary bead sets which are distinguishable under flow cytometry: each bead set is coated with a specific capture antibody, and fluorescence or streptavidin-labelled detection antibodies bind to the specific cytokine-capture antibody complex on the bead set.

Table 1 Clinical and demographic characteristics by treatment group (preaugmentation phase)

\begin{tabular}{|c|c|c|c|c|c|c|c|c|}
\hline \multirow{2}{*}{$\begin{array}{l}\text { Variables } \\
\text { Age mean minimum-maximum }\end{array}$} & \multicolumn{4}{|c|}{$\begin{array}{l}\text { Celecoxib augmentation } \\
\qquad(n=18)\end{array}$} & \multicolumn{4}{|c|}{$\begin{array}{l}\text { Treatment as usual } \\
\qquad(n=9)\end{array}$} \\
\hline & \multicolumn{4}{|c|}{$3320-60$} & \multicolumn{4}{|c|}{$31.7720-56$} \\
\hline Sex & Freque & ency & Perc & ent & Freque & ncy & Perc & cent \\
\hline Men & 5 & & 27 & & 4 & & & 4.4 \\
\hline Women & 13 & & 72 & & 5 & & & 5.6 \\
\hline Total & 18 & & 100 & 0.0 & 9 & & 10 & 0.0 \\
\hline Diagnoses & \multicolumn{4}{|c|}{ Frequency } & \multicolumn{4}{|c|}{ Frequency } \\
\hline Panic disorder & \multicolumn{4}{|c|}{1} & \multicolumn{4}{|c|}{1} \\
\hline GAD & \multicolumn{4}{|c|}{4} & \multicolumn{4}{|c|}{5} \\
\hline $\begin{array}{l}\text { Panic disorder with ago- } \\
\text { raphobia }\end{array}$ & \multicolumn{4}{|c|}{6} & \multicolumn{4}{|c|}{1} \\
\hline Social phobia & \multicolumn{4}{|c|}{5} & \multicolumn{4}{|c|}{2} \\
\hline OCD & \multicolumn{4}{|c|}{2} & \\
\hline Total & \multicolumn{4}{|c|}{18} & \multicolumn{4}{|c|}{9} \\
\hline Antidepressant treatment & \multicolumn{4}{|c|}{ Frequency } & \multicolumn{4}{|c|}{ Frequency } \\
\hline SSRI & \multirow{2}{*}{\multicolumn{4}{|c|}{10}} & \multicolumn{4}{|c|}{5} \\
\hline SNRI & & \multicolumn{3}{|c|}{4} & \multicolumn{4}{|c|}{2} \\
\hline NASSA & \multicolumn{4}{|c|}{3} & & & & \\
\hline$\beta$-blocker & \multicolumn{4}{|c|}{1} & \multirow{2}{*}{\multicolumn{4}{|c|}{2}} \\
\hline CBT & \multirow{2}{*}{\multicolumn{4}{|c|}{18}} & & & & \\
\hline Total & & & & & \multicolumn{4}{|c|}{9} \\
\hline $\begin{array}{l}\text { Week } 6 \text { psychometric mean } \\
\text { scores }\end{array}$ & Mean & $N$ & SD & SEM & Mean & $N$ & SD & SEM \\
\hline WEMWBS & 33.22 & 18 & 6.151 & 1.45 & 36.89 & 9 & 16.42 & 5.473 \\
\hline HADS-A W6 & 14.11 & 18 & 3.513 & .8328 & 812.11 & 9 & 5.819 & 1.94 \\
\hline HADS-D W6 & 10.17 & 18 & 3.682 & .8768 & 310.22 & 9 & 4.711 & 1.57 \\
\hline CGI-S W6 & 4.28 & 18 & 1.1327 & .2766 & 3.78 & 9 & 1.481 & 0494 \\
\hline OQUESA-GR W6 & 15.18 & 17 & 4.142 & 1.005 & 5 17.75 & 8 & 3.732 & 1.319 \\
\hline OQUESA-RP W6 & 18.89 & 18 & 5.2768 & 1.242 & 218 & 8 & 6.188 & 2.188 \\
\hline OQUESA-ED W6 & 10.39 & 18 & 4.0217 & .9547 & 710.38 & 8 & 5.975 & 2.112 \\
\hline OQUESA-NC W6 & 15.78 & 18 & 4.360 & 1.032 & 214.5 & 8 & 5.952 & 2.104 \\
\hline OQUESA-AC W6 & 15.83 & 18 & 6.653 & 1.576 & 610.17 & 6 & 3.817 & 1.558 \\
\hline
\end{tabular}

CBT, cognitive behavioural therapy; CGI-S, clinical global impression of illness severity; GAD, generalised anxiety disorder; HADS, hospital anxiety and depression scale; NASSA, noradrenergic and specific serotonergic antidepressant OCD, obsessive-compulsive disorder; OQUESA, Oxford questionnaire of emotional side effects of antidepressants; SNRI, serotonin-noradrenaline reuptake inhibitor; SSRI, selective serotonin reuptake inhibitor; WEMWEBS, Warwick-Edinburgh mental well-being scale.
Multiple cytokines can be recognized and measured by the differences in both bead sets, with chromogenic or fluorogenic emissions detected using flow cytometric analysis. We used a commercially available proinflammatory panel 1 (human) kit (from V-PLEX, MESO SCALE DISCOVERY, Rockville, Maryland, USA) to measure IL-1 $\beta$; IL-2; IL-4; IL-6; IL-8; IL-10; IL-12p70; IL-13; and TNF- $\alpha$ in the same sample.

Investigations of hair cortisol concentration (HCG) in this sample have been published previously (Elnazer et al., 2020). As celecoxib may affect cortisol concentration in vitro and in vivo (Liu et al., 2016), and because the interplay between inflammatory and neuroendocrine mechanisms modulates the response to stressors (McEwen et al., 1997; Turnbull et al., 1999; Elenkov et al., 2002; Elenkov, 2008; Wolkow et al., 2015), we examined correlations between HCC and cytokines.

\section{Ethical and research governance considerations}

The study was approved by Hampshire Research Ethics Committee (REC reference:16/SC/0038); the UK Health Research Authority (IRAS project ID: 170365); and the Medicinal Health Research Authority (EudraCT number:2016-000337-48), as part of a larger project investigating the potential influence of neuroinflammatory and endocrine factors in patients with anxiety disorders.

\section{Results}

A total of 170 referrals were received from primary care or secondary care mental health services. Of these, 135 patients dropped out following the initial explanation of the study [53 males (39\%), 82 females (61\%): mean age 34 years]. Thirty-five participants consented to take part and completed the Baseline assessment. Two patients dropped out before the week 6 review, and six patients dropped out before the week 12 review. All patients who expressed interest in the open-label celecoxib augmentation were carefully screened against the exclusion criteria for augmentation, and none of them met the exclusion criteria. Eighteen patients underwent open-label augmentation with celecoxib, and nine patients completed 12 weeks of treatment as usual.

\section{Clinical characteristics at Baseline}

Baseline clinical characteristics for study participants are detailed elsewhere (Elnazer et al., 2020). The mean scores (and standard error of the means) on rating scales were as follows: WEMWBS 27 (1.81); HADS-A 15.42 (0.88); HADS-D 13 (0.82); CGI-1 4.56 (0.22); OQUESA-GR 15.5 (1.17); OQUESA-RP 22.18 (0.75); OQUESA-ED 15.09 (3.17); OQUESA-NG 18 (1.27). Mean plasma cytokine concentrations (and HCG) were found to be within normal concentration ranges apart from a low mean concentration of IL-12p70 $(0.11 \mathrm{pg} / \mathrm{mL}$, SEM 0.04: normal range $0.26-0.38 \mathrm{pg} / \mathrm{mL}$ ) and an elevated mean 


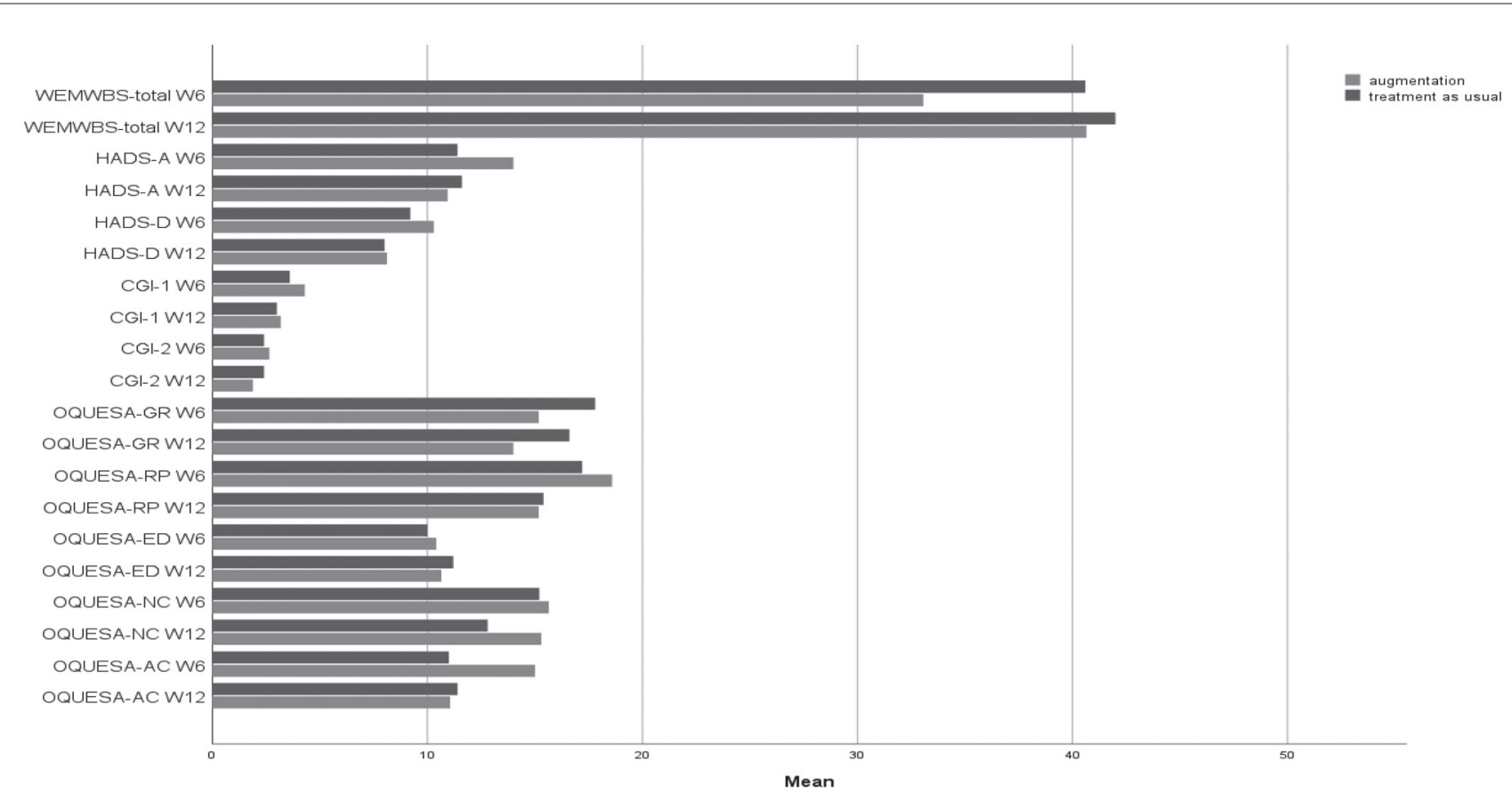

Rating scale scores at Week 6 and Week 12, by group.

concentration of TNF- $\alpha$ (2.37 pg/mL, SEM 0.43: normal range $0.10-1.75 \mathrm{pg} / \mathrm{mL}$ ).

\section{Preaugmentation phase}

Participants received 6 weeks of treatment as usual, from their usual clinician. Nineteen patients had undergone treatment with a selective serotonin reuptake inhibitor; six patients with a serotonin-noradrenaline reuptake inhibitor; three patients with a noradrenergic and specific serotonergic antidepressant; two patients with cognitive behavioural therapy; and a single patient with a $\beta$ blocker: two patients had not undergone any treatment by the week 6 review.

At week 6, participants remained troubled by anxiety and other symptoms HADS-A 13.70 (SEM 0.73); CGI-S 4.27 (SEM 0.23) CGI-I 2.69 (SEM 0.16) OQUESA-GR 16.30 (SEM 0.75); OQUESA-RP 18.97 (SEM 0.95); OQUESA-ED 11.06 (SEM 0.87) and OQUESA-NC 16.10 (SEM 0.86).

Analysis of cytokine (IFN- $\gamma$, IL-1 $\beta$, IL-10, IL-12p70, IL-13, IL-2, IL-4, IL-6, IL-8, TNF- $\alpha$ ) levels in participants who provided blood samples at week $6(n=25)$ found a low mean concentration of IL-2 [0.22 pg/mL (median $0.17 \mathrm{pg} / \mathrm{mL}$ ), normal range $0.22-2.68 \mathrm{pg} / \mathrm{mL}$ ] and a high mean concentration of TNF- $\alpha[2.16 \mathrm{pg} / \mathrm{mL}$ (median $1.60 \mathrm{pg} / \mathrm{mL}$ ), normal range $0.10-1.75 \mathrm{pg} / \mathrm{mL}]$. Paired sample $t$ test for cytokines concentrations found no significant changes from week 0 (Baseline) to week 6 .
Subgroup analyses found generally comparable scores ('Table 1). The treatment as usual group had a higher mean score of a general reduction in emotions (OQUESA-GR) (17.77 SEM 1.033) but lower scores on the scale for ascribing causality of emotional disturbance to antidepressants (OQUESA-AC) (12.27 SEM 1.966) when compared to the augmentation group (15.18 SEM 1.01) and (15.83 SEM 1.58), respectively.

Paired-T sample analysis showed no significant changes in rating scores from Baseline to week 6 , in both groups. No significant changes in inflammatory marker concentrations were seen in either group.

\section{Celecoxib augmentation phase}

By week 12, eighteen participants had undergone augmentation with celecoxib, and nine participants had continued 'treatment as usual'. Analysis using paired $t$ tests in the augmentation group found significant changes in mean scores from week 6 to 12 : WEMWBS 7.50 increase (SEM 2.63, $P=0.01$ ); HADS-A 3.17 reduction (SEM 0.52, $P=0.00)$; HADS-D 2.11 reduction (SEM 0.80, $P=0.02)$; CGI-S 1.11 reduction (SEM 0.18, $P=0.00$ ); CGI-I 0.78 reduction $(\mathrm{SEM} 0.32, P=0.03)$; OQUESA-RP 3.56 reduction (SEM 1.19, $P=0.01$ ) and OQUESA-AC 4.22 reduction (SEM 1.25, $P=0.00)$. Analysis using paired sample $t$ test in the treatment as usual group found changes in mean scores from week 6 to 12 as follows: WEMWBS 1.22 increase (SEM 2.10, $P=0.58$ ); HADS-A 0.11 increase (SEM 0.82, $P=0.90)$; HADS-D 0.78 decrease (SEM 1.11, 


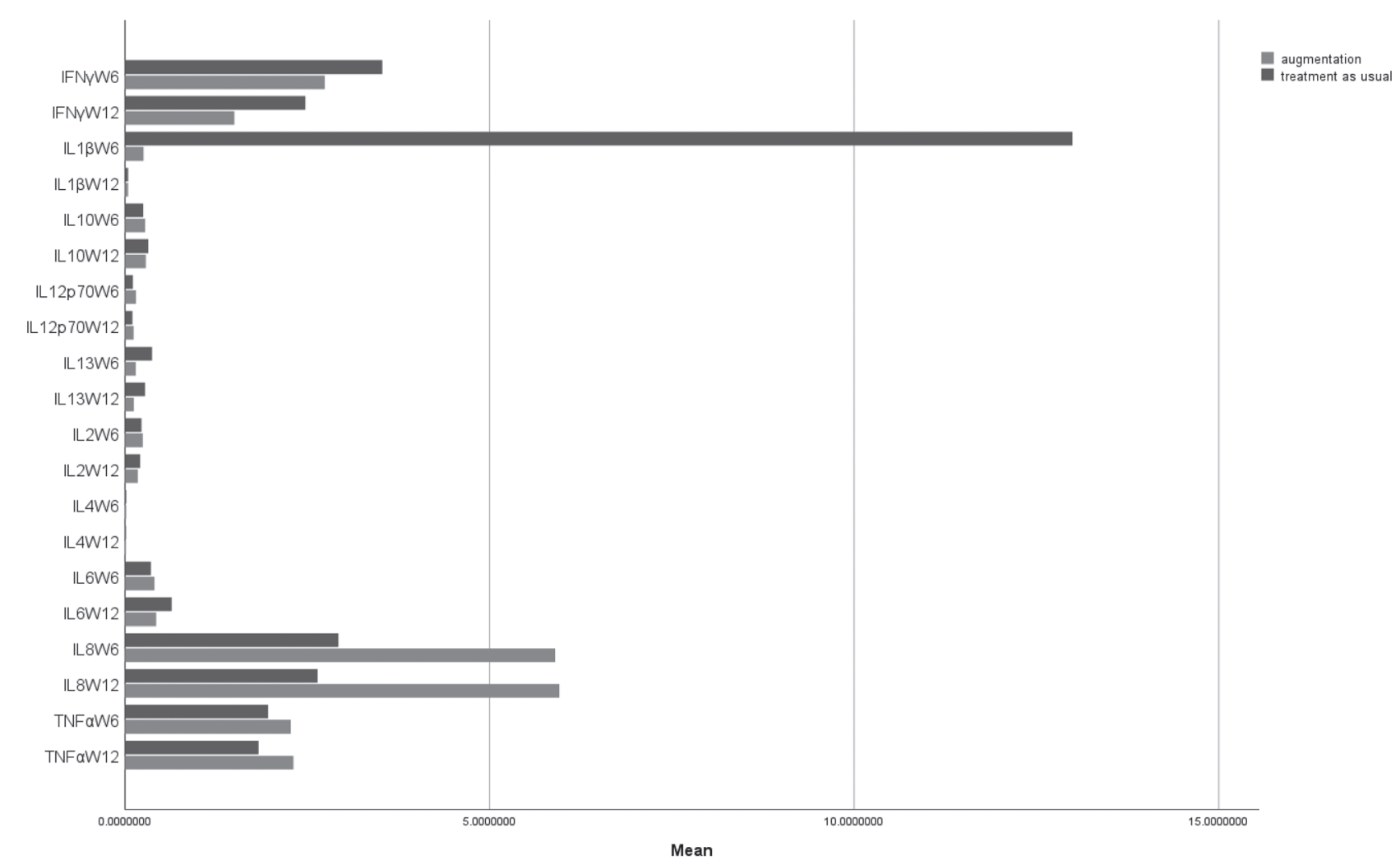

Cytokines' concentrations at Week 6 and Week 12 by group.

$P=0.50)$; CGI-S 0.33 decrease (SEM 0.28, $P=0.28$ ); CGI-I 0.44 increase (SEM $0.41, P=0.31$ ); OQUESA-GR 1.12 decrease (SEM 1.80, $P=0.55$ ); OQUESA-RP 2.00 decrease (SEM 0.82, $P=0.05$ ); OQUESA-ED no change (SEM 1.18, $P=1.00$ ); OQUESA-NC 1.87 decrease (SEM $1.09, P=0.13)$ and OQUESA-AC 0.40 increase (SEM 1.03, $P=0.72)($ Fig. 1).

Analysis of cytokine levels in the augmentation group found a low mean concentration of IL-1 $\beta(0.04 \mathrm{pg} / \mathrm{mL}$, SEM 0.024: normal range 0.11-24.3 pg/mL); low mean concentration of IL-12p70 (0.12 pg/mL, SEM 0.015: normal range $0.26-0.38 \mathrm{pg} / \mathrm{mL})$; low mean concentration of IL-13 (0.11 pg/mL, SEM 0.05: normal range 0.60-2.78 pg/ $\mathrm{mL})$ and a high mean concentration of TNF- $\alpha(2.33 \mathrm{pg} /$ $\mathrm{mL}$, SEM 0.21: normal range $0.10-1.75 \mathrm{pg} / \mathrm{mL})$. Paired sample $t$ testing found a significant change from week 6 to 12 in the mean concentration of IL-2 $0.06 \mathrm{pg} / \mathrm{mL}$ (SEM $0.02, P=0.00)$. Analysis of cytokines concentration in the treatment as usual group at week 12 found low mean concentrations of IL-1 $\beta(0.43 \mathrm{pg} / \mathrm{mL}$, SEM 0.018: normal range $0.11-24.3 \mathrm{pg} / \mathrm{mL})$; IL-12p70 (0.102 pg/mL, SEM 0.04: normal range $0.26-0.38 \mathrm{pg} / \mathrm{mL})$; IL-13 (0. $27 \mathrm{pg} /$ $\mathrm{mL}, \mathrm{SEM} 0.11$ : normal range $0.60-2.78 \mathrm{pg} / \mathrm{mL}$ ); and IL-2 (0.21 pg/mL, SEM 0.03: normal range 0.22-2.68 pg/mL): and a high mean concentration of TNF- $\alpha(2.33 \mathrm{pg} / \mathrm{mL}$ : normal range $0.10-1.75 \mathrm{pg} / \mathrm{mL}$ ). Paired sample $t$ testing, from week 6 to 12, found no significant change in this group (Fig. 2).

\section{Predicting response to treatment}

We used traditional forecasting models in an exploration of response to treatment. The analyses suggest some significant findings in the celecoxib augmentation group but not the treatment as usual group. Time series forecasting analysis, using autoregressive integrated moving average in the celecoxib augmentation group found significant predictability of emotional detachment scores (OQESA-ED) by HCC at week 6. Self-rated reduction in positive emotions at week 6 (OQUESA-RP) had significant predictability of depressive symptom severity (HADS-D), clinician-rated overall severity of illness (CGI-S) and self-rated reduction in positive emotions (OQUESA-RP) (Table 2).

\section{Discussion}

Patients with a diagnosis of any anxiety disorder (including OCD) at Baseline had low levels of IL-12p70 but high levels of TNF- $\alpha$. A significant correlation between HADS-A and IL-6 was found after adjusting for age, 
Table 2 Forecasting response to treatment, ARIMA model parameters ${ }^{a}$

\begin{tabular}{|c|c|c|c|c|c|c|c|c|}
\hline Outcome & Predictors & & & & Estimate & SE & $t$ & Sig. \\
\hline \multirow[t]{3}{*}{ HADS-A W12 } & \multirow[t]{2}{*}{ WEMWBS-total W6 } & \multirow[t]{2}{*}{ No transformation } & \multirow[t]{2}{*}{ Numerator } & Lag 0 & -0.332 & 0.078 & -4.273 & 0.001 \\
\hline & & & & $\operatorname{Lag} 3$ & -0.353 & 0.086 & -4.086 & 0.002 \\
\hline & HADS-A W6 & No transformation & Numerator & Lag 0 & 0.681 & 0.096 & 7.075 & 0.000 \\
\hline HADS-D W12 & OQUESA-RP W6 & No transformation & Numerator & Lag 0 & 0.421 & 0.033 & 12.661 & 0.000 \\
\hline \multirow[t]{4}{*}{ CGI-1 W12 } & \multirow[t]{2}{*}{ CGI-1 W6 } & \multirow[t]{2}{*}{ No transformation } & \multirow[t]{2}{*}{ Numerator } & Lag 0 & 0.740 & 0.096 & 7.739 & 0.000 \\
\hline & & & & Lag 2 & -0.342 & 0.102 & -3.358 & 0.006 \\
\hline & \multirow[t]{2}{*}{ OQUESA-RP W6 } & \multirow[t]{2}{*}{ No transformation } & Delay & & 3 & & & \\
\hline & & & Numerator & $\operatorname{Lag} 0$ & -0.081 & 0.029 & -2.826 & 0.015 \\
\hline \multirow[t]{3}{*}{ OQUESA-RP W12 } & \multirow[t]{3}{*}{ OQUESA-RP W6 } & \multirow[t]{3}{*}{ No transformation } & Delay & & 2 & & & \\
\hline & & & Numerator & Lag 0 & 0.385 & 0.134 & 2.860 & 0.019 \\
\hline & & & & $\operatorname{Lag} 5$ & -0.528 & 0.140 & -3.773 & 0.004 \\
\hline \multirow[t]{2}{*}{ OQUESA-ED W12 } & \multirow{2}{*}{$\begin{array}{c}\text { OQUESA-ED W12 } \\
\text { HCC W6 }\end{array}$} & No transformation & Constant & & 8.334 & 1.307 & 6.375 & 0.000 \\
\hline & & No transformation & Numerator & $\operatorname{Lag} 0$ & 0.332 & 0.144 & 2.298 & 0.035 \\
\hline
\end{tabular}

CGI-S, clinical global impression of illness severity; HADS, hospital anxiety and depression scale; HCC, hair cortisol concentration; OQUESA, Oxford questionnaire of emotional side effects of antidepressants; WEMWEBS, Warwick-Edinburgh mental well-being scale. ${ }^{2}$ For some models, some predictor series are not considered by expert modeller, due to missing values found in the estimation period.

diagnosis and sex. At week 6, mean scores on the HADS-A 13.70 (SEM 0.73) indicate that participating patients were still troubled by substantial symptoms. Analysis of inflammatory markers found low levels of IL-2 but high levels of TNF- $\alpha$, but the longitudinal analysis found no significant changes in inflammatory markers from Baseline to week 6.

At week 12, there was a significant change in questionnaire scores in the celecoxib augmentation group, with improved mental well-being (WEMWBS); reduced anxiety (HADS-A); reduced depression (HADS-D); lesser reduction in positive emotions (OQUESA-RP); and lesser attribution of antidepressants as cause (OQUESA-AC). There were no significant changes in questionnaire scores in the treatment as usual group. Nor were there significant changes in inflammatory marker concentrations in either group. The exploratory findings suggest that HCC, emotional detachment and reduction of positive emotions at week 6 might predict a positive response to 6 weeks' augmentation with celecoxib.

\section{Limitations}

The study sample had a high attrition rate. Nearly $79 \%$ of patients who expressed initial agreement to take part had dropped out before Baseline assessment. As a small number of participants consented to take part and were assessed with a large number of outcome measures, it was not possible to conduct further analyses of the findings. The augmentation group had double the number of patients compared to the treatment as usual group, as more participants preferred to opt for the augmentation group.

\section{Conclusion}

There was a significant change in questionnaire scores in the celecoxib augmentation group, reflecting improved mental well-being, reduced anxiety, reduced depression, lesser reduction in positive emotions and lesser attribution of antidepressants as the cause of altered emotions. Potential weaknesses of this study include the small sample size, the lack of randomisation and the lack of blindness to treatment group allocation. Our findings suggest that further studies, using a more robust double-blind randomised placebo-controlled design, should be undertaken to explore these preliminary results in greater detail.

\section{Acknowledgements}

The authors meet the criteria of authorship described by The International Committee of Medical Journal Editors (ICMJE).

The authors assert that all procedures contributing to this work comply with the ethical standards of the relevant national and institutional committees on human experimentation and with the Helsinki Declaration of 1975, as revised in 2008 .

\section{Conflicts of interest}

There are no conflicts of interest.

\section{References}

Abbasi SH, Hosseini F, Modabbernia A, Ashrafi M, Akhondzadeh S (2012). Effect of celecoxib add-on treatment on symptoms and serum IL-6 concentrations in patients with major depressive disorder: randomized double-blind placebo-controlled study. J Affect Disord 141:308-314.

Akhondzadeh S, Jafari S, Raisi F, Nasehi AA, Ghoreishi A, Salehi B, et al. (2009). Clinical trial of adjunctive celecoxib treatment in patients with major depression: a double blind and placebo controlled trial. Depress Anxiety 26:607-611.

American Psychiatric Association (2013). Diagnostic and statistical manual of mental disorders (DSM-5). Washington, DC: American Psychiatric Publishing.

Baune BT, Smith E, Reppermund S, Air T, Samaras K, Lux O, et al. (2012). Inflammatory biomarkers predict depressive, but not anxiety symptoms during aging: the prospective Sydney Memory and Aging Study. Psychoneuroendocrinology 37:1521-1530.

Carpenter LL, Heninger GR, McDougle CJ, Tyrka AR, Epperson CN, Price LH (2002). Cerebrospinal fluid interleukin-6 in obsessive-compulsive disorder and trichotillomania. Psychiatry Res 112:257-262.

Costello H, Gould RL, Abrol E, Howard R (2019). Systematic review and meta-analysis of the association between peripheral inflammatory cytokines and generalised anxiety disorder. BMJ Open 9:e027925.

Denys D, Fluitman S, Kavelaars A, Heijnen C, Westenberg H (2004). Decreased TNF-alpha and NK activity in obsessive-compulsive disorder. Psychoneuroendocrinology 29:945-952. 
Duivis HE, Vogelzangs N, Kupper N, de Jonge P, Penninx BW (2013). Differential association of somatic and cognitive symptoms of depression and anxiety with inflammation: findings from the Netherlands Study of Depression and Anxiety (NESDA). Psychoneuroendocrinology 38:1573-1585.

Elenkov IJ, Chrousos GP (2002). Stress hormones, proinflammatory and antiinflammatory cytokines, and autoimmunity. Ann N Y Acad Sci 966:290-303.

Elenkov IJ (2008). Neurohormonal-cytokine interactions: implications for inflammation, common human diseases and well-being. Neurochem Int 52:40-51.

Elnazer H, Lau L, Amaro H, Baldwin DS (2020). Hair cortisol concentration in anxiety disorders: exploration of relationships with symptom severity and inflammatory markers. Acta Neuropsychiatrica 1-7. doi:10.1017/neu.2020.35 [Epub ahead of print]

Fluitman S, Denys D, Vulink N, Schutters S, Heijnen C, Westenberg, H (2010). Lipopolysaccharide-induced cytokine production in obsessive-compulsive disorder and generalized social anxiety disorder. Psychiatry Res 178:313316.

Glaus J, von Känel R, Lasserre AM, Strippoli MF, Vandeleur CL, Castelao E, et al. (2018). The bidirectional relationship between anxiety disorders and circulating levels of inflammatory markers: results from a large longitudinal population-based study. Depress Anxiety 35:360-371.

Guy W (1976). ECDEU Assessment Manual for Psychopharmacology. Rockville, MD: National Institute of Mental Health. pp. 217-222, 313-331.

Halaris A, Cantos A, Johnson K, Hakimi M, Sinacore J (2020). Modulation of the inflammatory response benefits treatment-resistant bipolar depression: a randomized clinical trial. J Affect Disord 261:145-152.

Harden LM, Kent S, Pittman QJ, Roth J (2015). Fever and sickness behavior: friend or foe? Brain Behav Immun 50:322-333.

Hoge EA, Brandstetter K, Moshier S, Pollack MH, Wong KK, Simon NM (2009). Broad spectrum of cytokine abnormalities in panic disorder and posttraumatic stress disorder. Depress Anxiety 26:447-455.

Husain MI, Strawbridge R, Stokes PR, Young AH (2017). Anti-inflammatory treatments for mood disorders: systematic review and meta-analysis. Psychopharmacol 31:1137-1148.

lyengar RL, Gandhi S, Aneja A, Thorpe K, Razzouk L, Greenberg J, et al. (2013). NSAIDs are associated with lower depression scores in patients with osteoarthritis. Am J Med 126:1017.e11-1017.e18.

Janelidze S, Suchankova P, Ekman A, Erhardt S, Sellgren C, Samuelsson M, et al. (2015). Low IL-8 is associated with anxiety in suicidal patients: genetic variation and decreased protein levels. Acta Psychiatr Scand 131:269278.

Kandel ER, Schwartz JH, Jessell TM (2012). Principles of Neural Science. 5th ed. New York: McGraw-Hill, Health Professions Division.

Koh KB, Lee Y (2004). Reduced anxiety level by therapeutic interventions and cell-mediated immunity in panic disorder patients. Psychother Psychosom 73:286-292.

Konuk N, Tekin IO, Ozturk U, Atik L, Atasoy N, Bektas S, Erdogan A (2007). Plasma levels of tumor necrosis factor-alpha and interleukin-6 in obsessive compulsive disorder. Mediators Inflamm 2007:65704.

Liu S, Saloustros E, Berthon A, Starost MF, Sahut-Barnola I, Salpea P, et al. (2016). Celecoxib reduces glucocorticoids in vitro and in a mouse mode with adrenocortical hyperplasia. Endocr Relat Cancer 23:15-25.

Maes M, Berk M, Goehler L, Song C, Anderson G, Gałecki P, Leonard B (2012). Depression and sickness behavior are Janus-faced responses to shared inflammatory pathways. BMC Med 10:66.
Martin S, Vincent A, Taylor AW, Atlantis E, Jenkins A, Januszewski A, et al. (2015). Lower urinary tract symptoms, depression, anxiety and systemic inflammatory factors in men: a population-based cohort study. PLoS One 10:e0137903.

McEwen BS, Biron CA, Brunson KW, Bulloch K, Chambers WH, Dhabhar FS, et al. (1997). The role of adrenocorticoids as modulators of immune function in health and disease: neural, endocrine and immune interactions. Brain Res Brain Res Rev 23:79-133.

Monteleone P, Catapano F, Fabrazzo M, Tortorella A, Maj M (1998). Decreased blood levels of tumor necrosis factor-alpha in patients with obsessive-compulsive disorder. Neuropsychobiology 37:182-185.

Nery FG, Monkul ES, Hatch JP, Fonseca M, Zunta-Soares GB, Frey BN, et al. (2008). Celecoxib as an adjunct in the treatment of depressive or mixed episodes of bipolar disorder: a double-blind, randomized, placebo-controlled study. Hum Psychopharmacol 23:87-94.

Price J, Cole V, Doll H, Goodwin GM (2012). The Oxford Questionnaire on the Emotional Side-effects of Antidepressants (OQuESA): development, validity, reliability and sensitivity to change. J Affect Disord 140:66-74.

Priya PK, Rajappa M, Kattimani S, Mohanraj PS, Revathy G (2016). Association of neurotrophins, inflammation and stress with suicide risk in young adults. Clin Chim Acta 457:41-45.

Rapaport MH, Stein MB (1994). Serum cytokine and soluble interleukin-2 receptors in patients with panic disorder. Anxiety 1:22-25.

Sheehan DV, Lecrubier Y, Sheehan KH, Amorim P, Janavs J, Weiller E, et al. (1998). The Mini-International Neuropsychiatric Interview (M.I.N.I.): the development and validation of a structured diagnostic psychiatric interview for DSM-IV and ICD-10. J Clin Psychiatry 59 (Suppl 20):22-33;quiz 34.

Tennant R, Hiller L, Fishwick R, Platt S, Joseph S, Weich S, et al. (2007). The Warwick-Edinburgh Mental Well-being Scale (WEMWBS): development and UK validation. Health Qual Life Outcomes 5:63.

Turnbull AV, Rivier CL (1999). Regulation of the hypothalamic-pituitary-adrenal axis by cytokines: actions and mechanisms of action. Physiol Rev 79:1-71.

Vieira ML, Hamada RY, Gonzaga NI, Bacchi AD, Barbieri M, Moreira EG, et al. 2013). Could maternal exposure to the antidepressants fluoxetine and St John's Wort induce long-term reproductive effects on male rats? Reprod Toxicol 35:102-107.

Virtanen M, Shipley MJ, Batty GD, Hamer M, Allan CL, Lowe GD, et al. (2015). Interleukin-6 as a predictor of symptom resolution in psychological distress: a cohort study. Psychol Med 45:2137-2144.

Vogelzangs N, Beekman AT, de Jonge P, Penninx BW (2013). Anxiety disorders and inflammation in a large adult cohort. Trans/ Psychiatry 3:e249.

Wagner EY, Wagner JT, Glaus J, Vandeleur CL, Castelao E, Strippoli MP, et al. 2015). Evidence for chronic low-grade systemic inflammation in individuals with agoraphobia from a population-based prospective study. PLoS One 10:e0123757.

Wolkow A, Aisbett B, Reynolds J, Ferguson SA, Main LC (2015). Relationships between inflammatory cytokine and cortisol responses in firefighters exposed to simulated wildfire suppression work and sleep restriction. Physiol Rep 3:e12604.

Zigmond AS, Snaith RP (1983). The hospital anxiety and depression scale. Acta Psychiatr Scand 67:361-370.

Zorrilla EP, Redei E, DeRubeis RJ (1994). Reduced cytokine levels and T-cel function in healthy males: relation to individual differences in subclinical anxiety. Brain Behav Immun 8:293-312. 